\title{
El CLIMA ORGANIZACIONAL Y LA GESTIÓN INSTITUCIONAL
}

\section{EN LA FACULTAD DE EDUCACIÓN SEDE CANAS DE LA UNIVERSIDAD NACIONAL DESAN ANTONIO ABAD DEL CUSCO - 2011}

Lucila Olivares Torres ${ }^{1}$, Moisés Rodríguez Álvarez², Jesahel Vildoso Villegas ${ }^{3}$.

\begin{abstract}
RESUMEN
El propósito del trabajo de investigación fue determinar la relación existente entre el clima organizacional y la gestión institucional en la Facultad de Educación Sede Canas de la Universidad Nacional de San Antonio Abad del Cusco en el año 2011. El estudio fue de tipo descriptivo correlacional no causal, diseño no experimental, que permitieron determinar la relación existente entre las variables de estudio. La Hipótesis principal enunciaba la existencia de la relación directa entre el clima organizacional y la gestión institucional. Los resultados determinaron una relación directa, muy alta de $\mathrm{r}=0.94$ en docentes-administrativos y $\mathrm{r}=0.95$ en estudiantes, que afirmaron la relación entre las variables de estudio y consecuentemente aceptar la hipótesis planteada al inicio del estudio.

PALABRAS CLAVES: clima organizacional y gestión institucional.
\end{abstract}

\begin{abstract}
The purpose of the research work was to determine the existing relation between the organizational atmosphere and institutional management, in the Education Faculty in the Canas Headquarters of Cusco's San Antonio Abad National University, in 2011. The study was of the descriptive not causal type, with a non-experimental transversal descriptive and correlational design which allowed determining the relation existing between the study's variables. The Main Hypothesis enunciated the existence of a direct relationship between the organizational atmosphere and institutional management. The determination of the sample's size made possible through the non-probabilistic sampling constituted by 1 authority, 16 professors, 3 administrative staff members and 80 students who were applied an interview questionnaire and a survey structured in the Lickert Scale which were processed by
\end{abstract}

1 Doctora en Educación. Universidad Nacional de San Antonio Abad del Cusco. E-mail: luciolivarestorres@hotmail.com

2 Doctora en Educación. Universidad Nacional de San Antonio Abad del Cusco.

3 Doctora en Educación. Universidad Nacional Mayor de San Marcos. 
means of descriptive statistics and Pearson's correlation. The results determined a direct and very fortress relation of $\mathrm{r}=0.94$ among professors and administrative staff, and of $\mathrm{r}=0.95$ among students who affirmed the relation between the study's variables, and, consequently, accepted the hypothesis outlined at the beginning of the study.

KEYWORDS: Organizational atmosphere and institutional management.

$\mathrm{E}_{\mathrm{p}}^{\mathrm{l}}$ clima organizacional desde la percepción humana de la organización, es uno de los espacios fundamentales para el desarrollo de las organizaciones sobre todo para las educativas que incide en la estructura y dinámica del ambiente

\section{"Cómo los miembros integrantes de la comunidad universitaria perciban el clima y que éstos dispongan de un ambiente favorable con una sensación de libertad académica, posibilidades de crecimiento y realización personal.'}

organizacional, sin duda alguna, la organización no es únicamente la estructura física, sino, son las personas como eje de las relaciones humanas que en ella confluyen e interactúan. El clima es un indicador que mide el nivel de comunicación, es decir, el vínculo de confianza en las relaciones que existe entre sus miembros, los afectos que movilizan en el desempeño de las tareas y el grado de acuerdo sobre los criterios de decisión; que se reflejan en las actitudes, las motivaciones y el sentimiento de identidad institucional.
Para que una organización universitaria como institución académica orientada a la investigación y a la docencia cumpla con sus fines y propósitos, depende de cómo los miembros integrantes de la comunidad universitaria perciban el clima y que éstos dispongan de un ambiente favorable con una sensación de libertad académica, posibilidades de crecimiento y realización personal. Permitiéndoles el desempeño de las tareas académicas con mayor grado de competencia profesional, de participación efectiva y reafirmación de la identidad.

En este sentido, Covey (2003) propone como una necesidad, considerar a las personas en la organización "exactamente como queremos que ellos traten a nuestros clientes" Expresa que se puede comprar el trabajo de una persona, pero no se puede comprar su corazón ni su mente; en el corazón están su lealtad y su entusiasmo y en su mente, su creatividad, su ingenio y sus recursos intelectuales.

El clima organizacional constituye una de las estrategias de la gestión para reorientar los objetivos que se plantearon y que por alguna razón no se lograron en el plan estratégico institucional.

\section{METODOLOGÍA DE LA INVESTIGACIÓN}

La presente investigación es de tipo descriptivo correlacional no causal y considerando las características de la investigación, es un estudio de caso; cuyo objetivo es hacer un análisis específico de la Facultad de Educación Sede Canas de la Universidad Nacional de San Antonio Abad del Cusco referente a la relación entre clima organizacional y gestión institucional, así como establecer la relación entre las dimensiones de la gestión institucional: dirección, planeamiento, organización y control con el clima organizacional. 
La prueba descriptiva-correlacional utilizó el siguiente esquema:

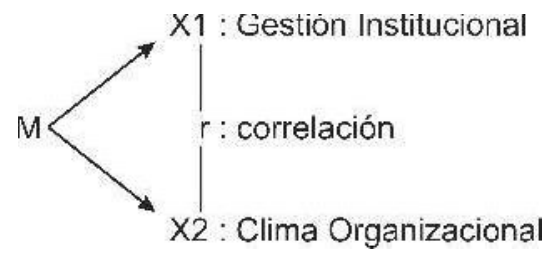

Para la contrastación de las hipótesis, se utilizó la prueba estadística correlacional, cuyo propósito es conocer la relación directa o no entre las variables clima organizacional y la gestión institucional.

La población estuvo conformada por 163 estudiantes, 16 docentes, 03 administrativos y 01directivo. Y la muestra estuvo constituida por 80 estudiantes, 16 docentes, 03 administrativos y 01 directivo.

La recolección de los datos se realizó a través de un cuestionario estructurado con una escala de tipo Lickert, de tres categorías de respuesta, el cual fue validado por juicio de expertos.

La confiabilidad del instrumento se obtuvo de los resultados de la prueba piloto, aplicado a 19 docentes y 19 estudiantes de la Facultad. Posteriormente, se calculó el Coeficiente Alfa de Cronbach el cual dio como resultado 0,982 lo que denota una elevada consistencia interna.

\section{RESULTADOS Y DISCUSIONES}

El estudio determinó una correlación directa muy fuerte entre el clima organizacional y la gestión institucional en la Facultad de Educación Sede Canas de la Universidad Nacional de San Antonio Abad del Cusco en el año 2011(según figura $\mathrm{N}^{\circ} 1$ y 2$) .(\mathrm{r}=0,94)$ en el análisis realizado a los docentes - administrativos; y de los estudiantes $(\mathrm{r}=0,95)$. Resultado que nos permitió aceptar la $\mathrm{H} 1$ y afirmar que a medida en que mejore el clima organizacional dentro de la Facultad ésta mejorará correlativamente la gestión institucional.
Figura 1. Correlación entre Clima Organizacional y Gestión Institucional: docentes - administrativos.

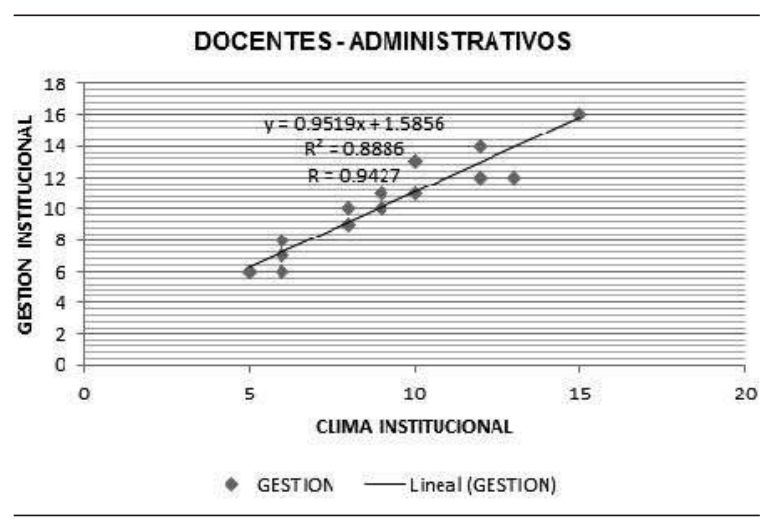

Figura 2. Correlación entre Clima Organizacional y Gestión Institucional: estudiantes

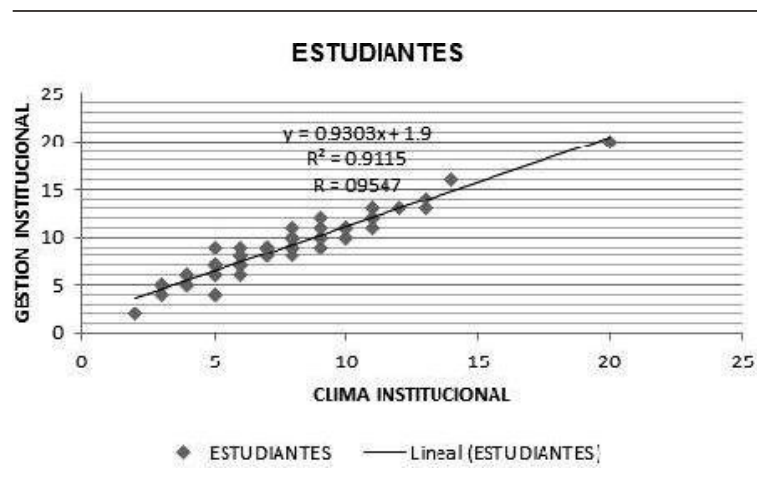

Asimismo con las hipótesis específicas se demostró la relación directa que existe entre las dimensiones de la gestión institucional con el clima organizacional, convirtiéndose en un indicador del funcionamiento institucional.

Al hacer comparaciones con algunas investigaciones relacionadas al tema de estudio, se encontró algunas coincidencias, como:

En el trabajo de Molocho Becerra (2010). "Influencia del clima organizacional en la gestión institucional de la sede administrativa UGEL $\mathrm{N}^{\circ} 01-$ Lima Sur - 2009". Se determinó que el clima institucional, expresado, en el potencial humano, en el diseño organizacional y en la cultura organizacional influye sobre la gestión institucional de la sede administrativa de la UGEL N ${ }^{\circ} 01$ Lima Sur. 
También, en el trabajo de Pelaes León (2010). "Relación entre el clima organizacional y la satisfacción del cliente en una empresa de servicios telefónicos". Se encontró la percepción de que existe relación directa entre el clima organizacional y la satisfacción del cliente en la empresa Telefónica del Perú a medida que mejoran las relaciones interpersonales mejora correlativamente la satisfacción del cliente.

Asimismo, en el trabajo de Arela Surco (2011) "Relación de clima

“Los estudios revisados
nos permiten reafirmar
que a medida que
mejore el clima
organizacional, mejorará
correlativamente la
gestión institucional.”

organizacional en la gestión institucional de las instituciones educativas estatales de nivel secundario del distrito de Ayaviri Melgar, Puno". Se demostró que existe una relación directa significativa entre el nivel de clima organizacional y gestión institucional en las instituciones educativas estatales de nivel secundario de Ayaviri Melgar, Puno.

El Clima es una variable que interviene entre los factores del sistema organizacional y el comportamiento individual. El clima organizacional se constituye cada vez más en un factor que refleja las facilidades o dificultades que encuentra el trabajador para aumentar o disminuir su productividad o para encontrar el punto de equilibrio. Al respecto Chiavenato (2009) manifiesta, que el clima organizacional influye en el estado motivacional de las personas y, a su vez, recibe influencia de éste: es como si hubiera una realimentación recíproca entre el estado motivacional y el clima organizacional. También Gonçalves (2000:31) señala, que el clima organizacional influye en el comportamiento manifiesto de los miembros, a través de percepciones estabilizadas que filtran la realidad y condicionan los niveles de motivación laboral y rendimiento profesional. Igualmente, Sandoval (2004) indica, que el clima organizacional es el ambiente de trabajo, percibido por los miembros de la organización y que incluye estructura, estilo de liderazgo, comunicación, motivación y recompensas, todo ello ejerce influencia directa en el comportamiento y desempeño de los individuos.

Los estudios revisados nos permiten reafirmar que a medida que mejore el clima organizacional, mejorará correlativamente la gestión institucional. Un Clima Organizacional estable y favorable, es una inversión a largo plazo.

\section{CONCLUSIONES}

Existe una relación entre el clima organizacional y la gestión institucional de la Facultad de Educación Sede Canas de la Universidad Nacional de San Antonio Abad del Cusco, en el año 2011, con un índice de correlación 0,94 en docentes - administrativos y 0,95 en estudiantes que determina una correlación positiva muy fuerte. 
Existe relación entre la dimensión dirección y el clima organizacional en la Facultad de Educación Sede Canas de la Universidad Nacional de San Antonio Abad del Cusco, en el año

2011 , con un índice de correlación $\mathrm{r}=0.70$ en docentes- administrativos y $r=0,77$ en estudiantes que determina una correlación positiva considerable.

Existe relación entre la dimensión planeamiento y el clima organizacional en la Facultad de Educación Sede Canas de la Universidad Nacional de San Antonio Abad del Cusco, en el año 2011, con un índice de correlación $\mathrm{r}=0.62$ en docentes-administrativos y $\mathrm{r}=0,66$ en estudiantes que determina una correlación positiva considerable.

Existe relación entre la dimensión organizacional y el clima organizacional de la Facultad de Educación Sede Canas de la Universidad Nacional de San Antonio Abad del Cusco, en el año 2011, con un índice de correlación $\mathrm{r}=0.41$ en docentes - administrativos que determina una correlación alta, directa y positiva y $r=0,37$ en estudiantes que determina una correlación positiva débil.

Existe relación entre la dimensión control y el clima organizacional de la Facultad de Educación Sede Canas de la Universidad Nacional de San Antonio Abad del Cusco, en el año 2011 , con un índice de correlación $\mathrm{r}=0.21$ en docentes- administrativos y $\mathrm{r}=0,37$ en estudiantes que determina una correlación positiva débil.

\section{REFERENCIAS}

Blejmar, B. (2005) Gestionar es hacer que las cosas sucedan.

Argentina: Ediciones Novedades Educativas S.A.

Brovelli, M. (2000) Nuevos viejos roles en la gestión educativa.

Argentina: Ediciones Homo Sapiens.

Covey, S. R. (2002) Los 7 hábitos de la gente altamente efectiva. México: Editorial Paidos.

Chiavenato, I. (2000) Introducción a la teoría general de la administración. (5a ed.). México: Mc Graw-Hill Interamericana Editores.

(2009) Comportamiento organizacional. McGraw-Hill Interamericana Editores S. A. México. (2009) Administración de recursos humanos. México: McGraw-Hill Interamericana Editores S. A

Drucker, P. (2002) La gerencia en la sociedad futura. Bogotá. Colombia: Editorial Norma

Espinoza, N. (2000) Gerencia universitaria: Lima Perú: Editorial San Marcos.

Falcón, Ernesto. Clima organizacional en aula, 2005 http://www.monografias .com/us uario/p erf iles/ falco_solis_ernesto, [consulta: 18 de mayo del 2011]

García, F. (2002) Organización escolar y gestión de centros educativos. Málaga España: Ediciones Aljibe S.L.

García, Inmaculada. La formación del clima psicológico y su relación con los estilos de liderazgo, 2006, essantabarbara.files.wordpress.com/.../tesis-laform acion-del-clima-psicolog... [Consulta: $22 \mathrm{de}$ marzo del 2012]

Gómez, Valentina. Estudio clima organizacional en la ESE hospital San José de Pereira, 2010, http://scielo.bvspsi. org.br/scielo.php?pid=S179499982006000200013 \& script $=$ sci arttext\&tlng $=$ es. Panorama sobre los estudios de clima organizacional en Bogotá, Colombia, [consulta: 20 de mayo del 2011]

Goncalves, A. (2000) Fundamentos del clima organizacional: sociedad latinoamericana para la calidad (SLC).

Ivancevich M. J. (2006) Comportamiento organizacional., (7a. ed.). México: Mc Graw Hill.

Lepeley, M.T. (2003) Gestión y calidad en educación. México: McGraw Hill.

Melinkoff, R. (2001) Los Procesos Administrativos. Caracas Venezuela: Editorial Panapo.

Mintzberg, H. (1991) Mintzberg y la dirección. Madrid España: Editorial Díaz de Santos S.A.

Molinar, M. del C. (2001). Liderazgo en la labor docente. México: Trillas.

Molocho, Nicanor. Influencia del clima organizacional en la gestión institucional de la sede administrativa UGEL $\mathrm{N}^{\mathrm{o}} 01 \quad$ Lima sur, 2009, http://www.cybertesis.edu.pe/ sisbib/2010/molocho bn/html/indexframes.html, [consulta: 07 de enero del 2011]

Pelaes, Oswaldo. Relación entre el clima organizacional y la satisfacción del cliente en una empresa de servicios telefónicos,

2010,http://www.cybertesis.edu.pe/sisbib/ 2010/pelaez lo/html/index-frames.html [consulta: 14 de enero del 2011]

Sánchez, Juan. La influencia de la gestión universitaria en el clima organizacional: un estudio comparativo de tres universidades, http://www.cybertesis.edu.pe/ sisbib/2005/sanchez sj/html/indexframes.html [consulta: 05 de febrero del 2011] 
Sandoval, María. Conceptos y Dimensiones del Clima Organizacional, 2004 www.publicaciones.ujat.mx/ publicacione s/.../08_ Ensayo_Dime nsiones.pdf, [consulta: 23 de diciembre del 2012]

Sengle, P. (2000) La danza del cambio. Bogotá Colombia: Editorial Norma S.A.

Sengle, P. (2003) La quinta disciplina. Barcelona España: Editorial Soler S.A 